\title{
Nonuniform Expression of Habituation in the Activity of Distinct Classes of Neurons in the Aplysia Abdominal Ganglion
}

\author{
Chun Xiao Falk, ${ }^{1}$ Jian-young Wu, ${ }^{1}$ Lawrence B. Cohen, ${ }^{1}$ and Akaysha C. Tang ${ }^{1,2, a}$ \\ 'Department of Cellular and Molecular Physiology, Yale University School of Medicine, New Haven, Connecticut 06510, \\ 2Department of Psychology, Harvard University, Cambridge, Massachusetts 02138, and the Marine Biological Laboratory, \\ Woods Hole, MA 02543
}

\begin{abstract}
Global observations of neuronal response in the Aplysia abdominal ganglion were made during habituation of the gill withdrawal reflex using voltage-sensitive dye recording. This technique makes it possible to measure the spike activity of $30-50 \%$ of the 1000 neurons present in the ganglion. Our experiments address the issue of how habituation is expressed in the activity of the population of neurons responding to siphon stimulation. Several classes of neurons exhibited characteristically distinct responses to the stimuli and to habituation training. One class of neurons (group I) responded to the onset and offset of the sensory stimulus although they are probably not primary sensory neurons. They habituate only partially when the behavioral reflex has already habituated completely. Two other classes (groups II and III) both have sustained responses to the touch, but habituate differently. Members of group III habituate completely while those in group II habituate only partially. Another class of neurons are inhibited by the stimulus (group IV). They become less inhibited after habituation. The response of both group I and group I! are new classes of response that have not been previously reported.
\end{abstract}

[Key words: habituation, gill withdrawal reflex, voltagesensitive dye, optical recording, Aplysia, neuron clustering]

The neuronal basis of the Aplysia gill withdrawal reflex and its modification by habituation, a simple form of learning, have been studied extensively (Castellucci et al., 1970; Kupfermann et al., 1970; Pinsker et al., 1970; Byrne, 1982; Goldberg and Lukowiak, 1984; Bailey and Chen, 1988). The cellular correlates of behavioral habituation were attributed largely to the "homosynaptic depression of the excitatory efficacy of the monosynaptic sensory-to-motor connection" in the abdominal ganglion (Dudai, 1989). Most studies have focused on the events at the site of plasticity that this model suggested. However, recent electrophysiological experiments suggest that the minimal neu-

Received Jan. 4, 1993; revised Apr. 5, 1993; accepted Apr. 6, 1993

We thank Ray Falk and Paul Katz for helpful discussions of data analysis and thank Barbara Ehrlich, Bill Ross, and Terry Walters for comments on the manuscript. We are grateful to David Senseman for the loan of the 464 element photodiode anray and the Silicon Graphics workstation. Vic Pantani and Henrik Abildgaard of the Physiology electronics shop designed and constructed the amplifiers and analog-to-digital converter used to record the output of the diode array. This work was supported in part by Grant NS08437 from NINDS, and an IBM and a William James Fellowship.

Correspondence should be addressed to Chun Falk, Department of Physiology, Yale University School of Medicine, 333 Cedar Street, New Haven, CT 06510.

"Present address: 2159 NW Irving, Portland, Oregon.

Copyright (c) 1993 Society for Neuroscience $0270-6474 / 93 / 134072-10 \$ 05.00 / 0$ ronal circuit for the reflex is more complex (Lukowiak, 1978; Hawkins et al., 1981; Frost et al., 1988; Trudeau and Castellucci, 1992). Indeed, optical measurements show that 200-300 neurons in the ganglion are activated by the sensory stimulus that initiates the gill withdrawal reflex (Zecevic et al., 1989; Nakashima et al., 1992). Because of this complexity, it is important to obtain a more global view of neuron activity during the process of habituation.

The LE siphon sensory neurons (Byrne et al., 1978a,b), previously thought to provide the sensory input for the gill withdrawal reflex, were recently reported to be activated after the motor neurons (Cohen et al., 1991). Thus, these neurons are probably not the major touch sensory neurons for the gill withdrawal reflex. Optical measurements may provide useful information about other sources of sensory input. This study addresses the question of how habituation is expressed in the response patterns of individual neurons in this population and how the change in these responses contributes to the behavioral change.

Preliminary reports have been published (Falk et al., 1991, 1992).

\section{Materials and Methods}

Preparation and procedures. Aplysia californica weighing 5-20 gm were obtained from Marinus (Long Beach, CA). At this stage, abdominal ganglia have about 900 neurons (Coggeshall, 1967; Cash and Carew, 1989). Experiments were carried out on the isolated siphon preparation (Fig. 1) developed by Kupfermann et al. (1971). The isolated siphon preparation was placed in a Lucite chamber with compartments for siphon, gill, and abdominal ganglion isolated by Vaseline seals. Except where specifically mentioned, the ganglion was bathed either in natural seawater or in an artificial seawater $(460 \mathrm{~mm} \mathrm{NaCl}, 10 \mathrm{~mm} \mathrm{KCl}, 11 \mathrm{~mm}$ $\mathrm{CaCl}_{2}, 55 \mathrm{mM} \mathrm{MgCl}_{2}$, and $10 \mathrm{~mm}$ Tris- $\mathrm{HCl}, \mathrm{pH}$ 7.6). The nondesheathed ganglion was stained with $0.1-0.15 \mathrm{mg} / \mathrm{ml}$ of the voltage-sensitive dye RH155 (Grinvald et al., 1982) (available as NK3041, Nippon Kankoh Shikiso Kenkyusho Co. Ltd., Okayama, Japan). The staining procedure, which consisted of four alternations of $2.5 \mathrm{~min}$ in dye and $2.5 \mathrm{~min}$ of wash, was designed to avoid pharmacological effects of the dye ( $\mathrm{Na}$ kashima et al., 1992). The staining was done before the ganglia were pinned out in the Sylgard chamber; staining after pinning out seemed to result in less complete optical recordings. Mechanical stimulation (2 $\mathrm{gm} / 600 \mathrm{msec}$ ) was delivered to the siphon skin via a computer-controlled motor moving a $0.6 \mathrm{~mm}$ glass rod with a rounded tip. The siphon was pinned down and bathed in a low-calcium, high-magnesium saline $\left(228 \mathrm{~mm} \mathrm{NaCl}, 10 \mathrm{~mm} \mathrm{KCl}, 1 \mathrm{mM} \mathrm{CaCl}_{2}, 220 \mathrm{~mm} \mathrm{MgCl}_{2}\right.$, and $10 \mathrm{~mm}$ Tris- $\mathrm{HCl}, \mathrm{pH}$ 7.6) to block synaptic transmission. This solution was used to block siphon movement in order to obtain a consistent stimulation site. Preliminary experiments indicated that this solution in the siphon chamber had no detectable effect either on the response of neurons in the ganglion to the mechanical stimulation or on the resulting gill withdrawal. The temperature was usually kept at $16 \pm 1{ }^{\circ} \mathrm{C}$.

The gill movements were recorded on videotape. The time course of the change in gill area was obtained from the videotape by electronically 


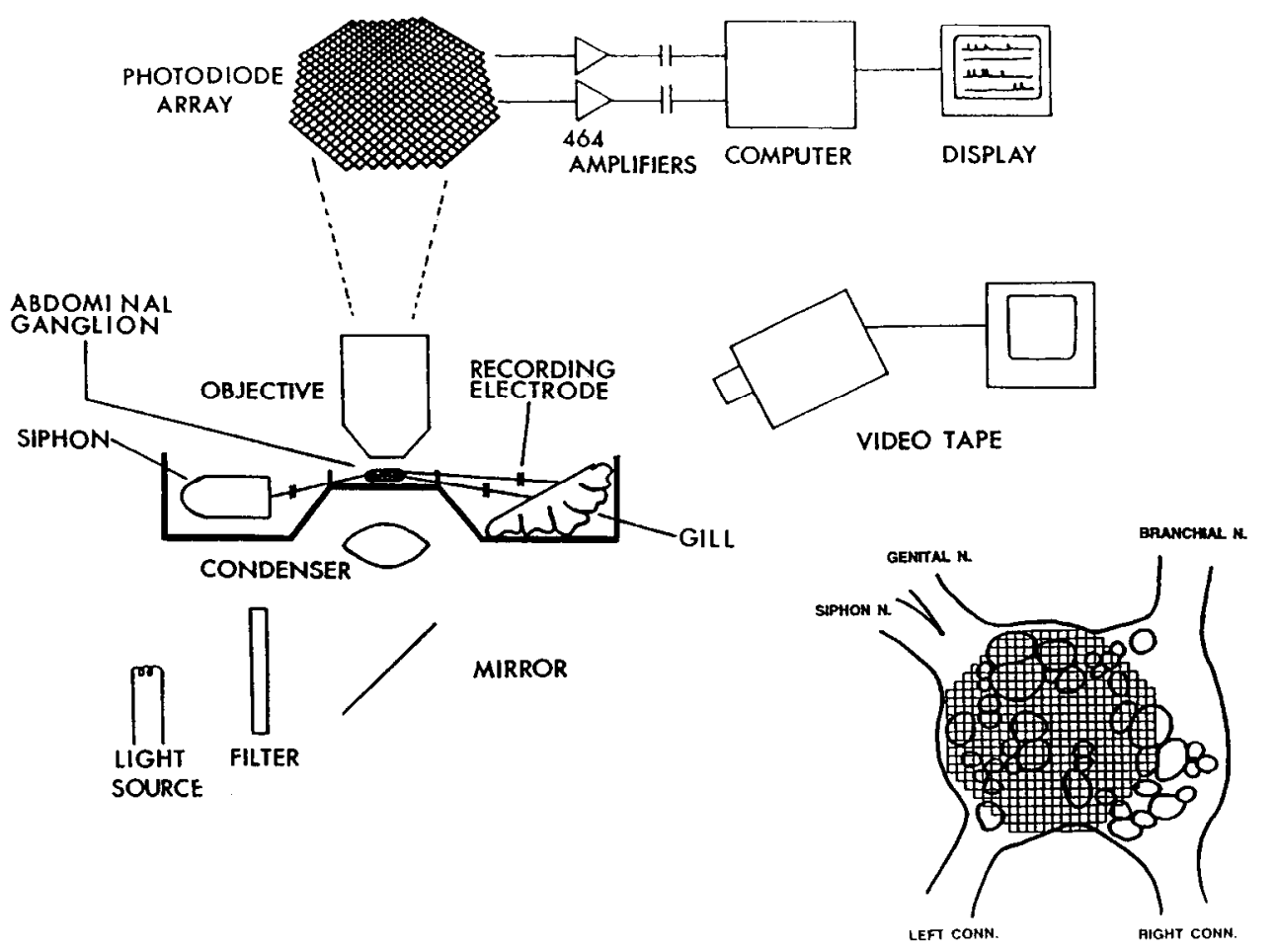

Figure 1. Schematic diagram of the apparatus. Light from a tungsten halogen lamp passed through a $705 \perp 25$ $\mathrm{nm}$ interference filter and was focused on the preparation. We used a modification of Kohler illumination (Leitz Ortholux II microscope) where the condenser iris was opened so that the condenser numerical aperture would equal the objective $(25 \times, 0.4 \mathrm{NA})$ numerical aperture. A photodiode array was placed at the plane where the objective forms the real, inverted image. The photodiode array detects the light transmitted by the ganglion; the outputs from each diode were individually amplified. The amplifier outputs were digitized by a 512 channel analog-to-digital converter and stored in a Motorola $68030 \mathrm{VME}$ bus computer. The relative size and position of the photodiode array and the image of an abdominal ganglion are shown on the lower right.

integrating the video signal. In the video frame the gill was brighter than the background. The video signal was filtered with a threshold discriminator to convert the brighter signals to ones and the darker signals to zeros. Integration of the ones results in the analog signal representing the area of the gill.

The microscope objective was focused at the middle of the ganglion in an effort to maximize the number of neurons detected in each recording. The objective projected this image onto a photodiode array situated at the image plane of the microscope objective. The relative position of the array and the image of a ganglion are shown schematically in the lower right of Figure 1. The absorption signals in the light transmitted by the abdominal ganglion were recorded by a photodiode array (124 elements of a $12 \times 12$ or a 464 element array; Centronic Inc., Newbury Park, CA). The output of each detector was individually amplified. A 4-pole switched-capacitance Bessel filter in the amplifiers set at a cutoff frequency of $200 \mathrm{~Hz}$ was used to limit the high-frequency noise. A single-pole RC filter with a cutoff frequency of $1.5 \mathrm{~Hz}$ was used to eliminate the DC offset and limit low-frequency noise. The photodiode amplifier outputs were multiplexed, digitized, and stored in a Motorola VME bus computer. Additional filtering was carried out digitally. Additional details of the apparatus can be found in Wu and Cohen (1993). We used a sampling rate of one data point per millisecond for the $12 \times 12$ array (recording time, $8 \mathrm{sec}$ ) and one point per $1.6 \mathrm{msec}$ for the 464 element array (recording time, $13 \mathrm{sec}$ ). There were 8192 data points for each detector, $2 \times 1024$ before the stimulus and $6 \times$ 1024 after the stimulus. A unit of 1024 data points is called a page.

The raw data from the optical recordings consist of cell body spike signals on photodiode outputs; further analysis is required to determine the activity of individual neurons from this data (for examples, see London et al., 1987; Zecevic et al., 1989). We used a semiautomatic program with the following steps. First, the operator chose an individual occurrence of spike signals on one (or more) detector(s) as a template. Using this template, the program determined the times of similar spike signals on these detectors and then displayed the template together with individual occurrences, allowing the operator to determine which of the individual occurrences should be grouped together as activity of one neuron. The program stored these spike times and locations and then erased these spike signals from the data. In this way the raw data became progressively simplified until the spike times of the neuron with the smallest acceptable signal-to-noise ratio were recorded. To analyze experiments with several recordings from one preparation, we first concatenated the raw data from all trials so that the analysis could be done as if it were one long recording. In this way we could follow the activity of individual neurons throughout all of the trials of one experiment. Because of variability in the number and position of ncurons from ganglion to ganglion we were not able to follow neurons across animals.

Data analysis. Two files were generated, a spike file that contained the times of the action potentials for the individual cells and a location file containing the diode elements that had the optical signals for those cells. Diodes with signals greater than $50 \%$ of the largest signal for each cell were included in the location file. The spike and location files were generated on the Motorola computer; further analysis of this data, used to generate Figures 2-8, was carried out using MATHEMATICA (Wolfram Research, Champaign, IL, version 2.1) on a Silicon Graphics Iris Indigo computer. The source codes for all programs are available from the authors.

Cluster analysis. In order to group neurons with distinctive response characteristics, a cluster analysis (Everitt, 1974) was employed using the software package sPss-x (SPSS Inc., Chicago, 1988). As input the program was given the firing rates (spike counts in each interval) during both control and habituation trials for each cell. We used intervals of 2048 data points. Each cell's response was described by an $n$-dimensional vector comprising the spike counts in $n$ successive time intervals. For the experiment illustrated in Figure 2 with eight trials, $n$ was 32 (4 $\times 8$ ). The clustering program then grouped the cells according to the distance between their vectors. With this input the results are more sensitive to the absolute spike counts in each interval than to response patterns. In order to separate the cells with small spike counts but obviously different response patterns, the program generated several groups among the cells that had similar response patterns but differed in spike counts. Recombination and adjustments were made using human pattern recognition to obtain the grouping of the neurons shown in Figure 2.

Experimental design. Three sets of experiments are reported: two sets of habituation experiments and one control set. In the first habituation set $(n=6)$, the interstimulus interval (ISI) was $1 \mathrm{~min}$ and 40 habituation trials were used. In the second set $(n=3)$, the ISI was $30 \mathrm{sec}$ and 80 habituation trials were used. The experiment whose results are illustrated in Figures $2-4$ is from the second set. From each preparation, only 8-10 optical recordings were made at selected stages of habituation since pilot experiments indicated that, by making no more than ten 8 $13 \mathrm{sec}$ optical recordings, photodynamic damage and dye bleaching could be avoided and normal recoveries could be achieved (see Fig. 6). In the ninc cxpcriments we analyzed, four trials that showed clear contamination with activity from spontaneous gill contractions were removed from further analysis. Additional experiments that showed no 


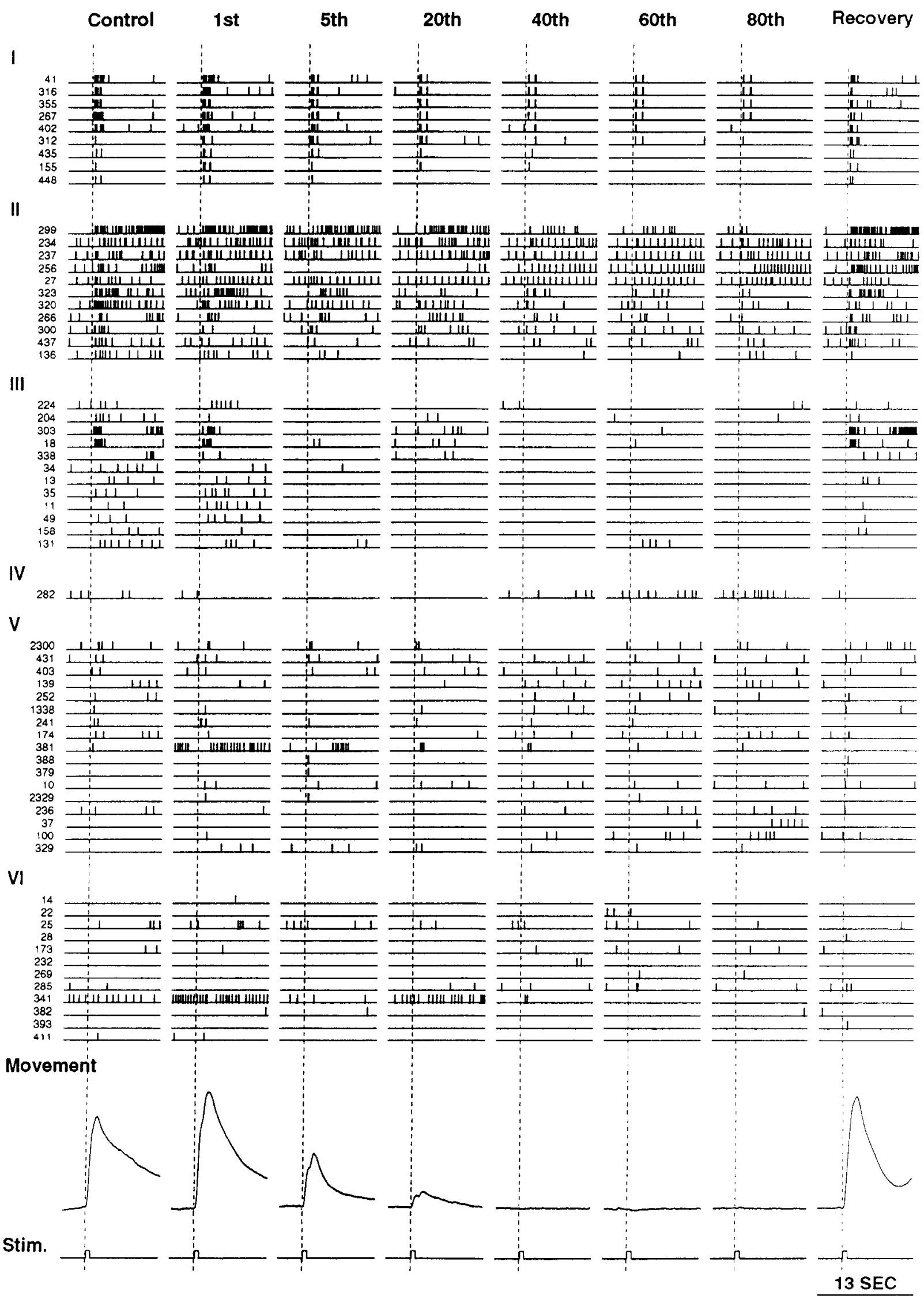


$\mathbf{B}$

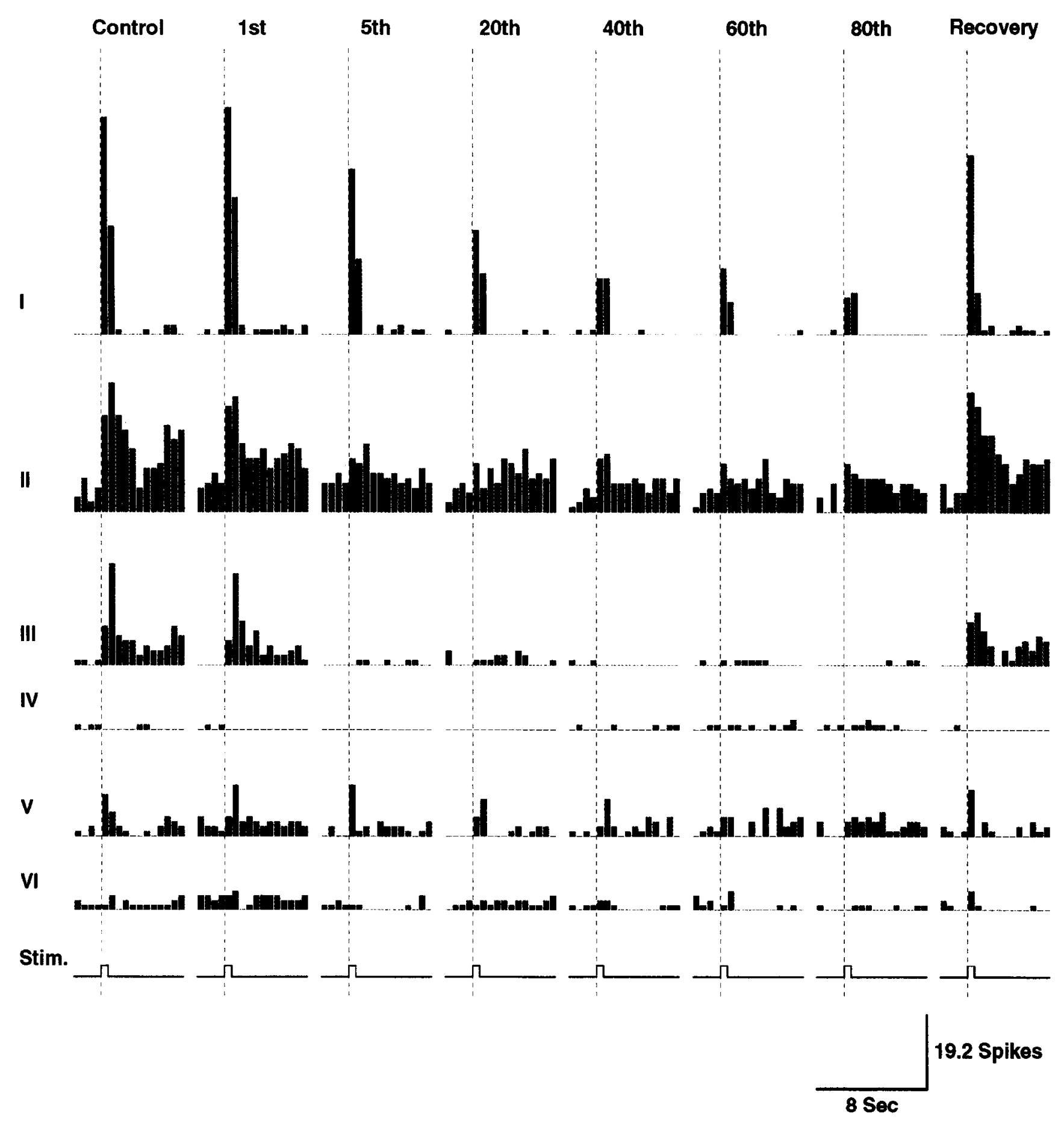

Figure 2. A, Raster diagrams of the action potential activity detected in an Aplysia abdominal ganglion in response to eight presentations of a light touch before, during, and after a habituating procedure. Each line represents one neuron and each vertical tick mark represents an action potential in that neuron. Each vertical panel is a $13 \mathrm{sec}$ optical recording. Recordings were made during the $1 \mathrm{st}$, 5th, 20th, 40th, 60th, and 80 th habituation trials, during a control trial $15 \mathrm{~min}$ before the first habituation stimulus, and during a recovery trial 40 min after the last habituation trial. The cells are arranged into six groups indicated by the roman numerals on the left. The small numbers on the left are identification numbers derived from the cell locations on the photodiode array. The gill contractions (bottom) were obtained by measuring the gill area from videotape recordings. The bottom trace shows the current steps that drove the mechanical toucher. The dashed lines indicate the beginning of these current steps. $B$, Histograms of spike density. Each histogram represents spike counts per $812 \mathrm{msec}$ summed over all the neurons of the corresponding groups in $A$. These histograms are not normalized for the number of neurons in each group. 


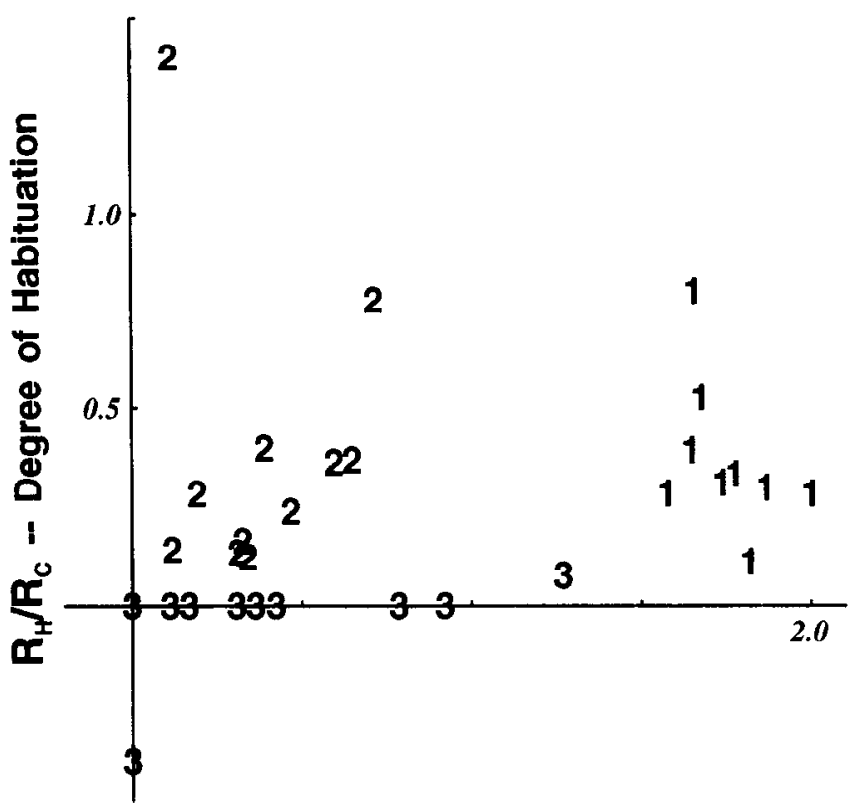

$\left(2 C_{3}-C_{1,2}\right) / C_{\text {total }}$-- Response Pattern

Figure 3. Visualization of the clustering of three groups of neurons from the experiment illustrated in Figure 2 in a two-dimensional plot. Each neuron is plotted as its group number. Although the neurons are not ideally clustered, cells in the three groups are separated. For the $\mathrm{x}$-axis, pooling across all trials, the spike count in the third page $\left(C_{3}\right.$, the first 1024 data points after the stimulus) is adjusted for the prestimulus spike count $\left(C_{1.2}\right)$, the spike count in the first two pages (2048 data points before the stimulus), and normalized by the total spike count $\left(C_{\text {total }}\right)$. This measure is sensitive to the response pattern: excited or inhibited, brief or sustained. For the y-axis, $R_{l} / R_{c}$ is sensitive to the degree of habituation; $R_{n}$ is the response of each cell in the habituated trials, and $R_{C}$. is the response of the same cell in the control and recovery trials. $R_{H}$ and $R_{C}$ were calculated as $C_{3,4}-C_{1.2}$, the spike count in the 2048 data points after the stimulus minus the spike count in the 2048 data points before the stimulus.

behavioral recovery from habituation or obvious bleaching in the optical recording were discarded. There were five experiments in the control set using an ISI of $15 \mathrm{~min}$ - an interval that did not lead to dramatic habituation of the gill withdrawal response. The control experiments were used to test for systematic effects of the optical recording procedure in the absence of habituation training.

\section{Results}

\section{Distinct classes of neurons}

We first extracted the action potential activity of individual neurons from the signals on the photodiode outputs. Then, with the guidance of cluster analysis, the neurons were divided into six groups based on the temporal pattern of their response across the control and habituation trials. The results for one experiment are displayed as a raster diagram (Fig. $2 A$ ) with the groups indicated by the roman numerals on the left. Each vertical panel is a $13 \mathrm{sec}$ optical recording. Each horizontal line represents one neuron and each vertical tick mark represents an action potential in that neuron. The magnitude of gill contraction measured from videotape recordings is shown at the bottom. The effect of habituation on the behavior is clear. The gill contraction is substantially decreased in the fifth habituation trial and has disappeared by the 40 th trial.

The corresponding spike density histograms of the six groups of neurons for each trial are shown in Figure $2 B$. These histo-

\section{Group I Neurons}

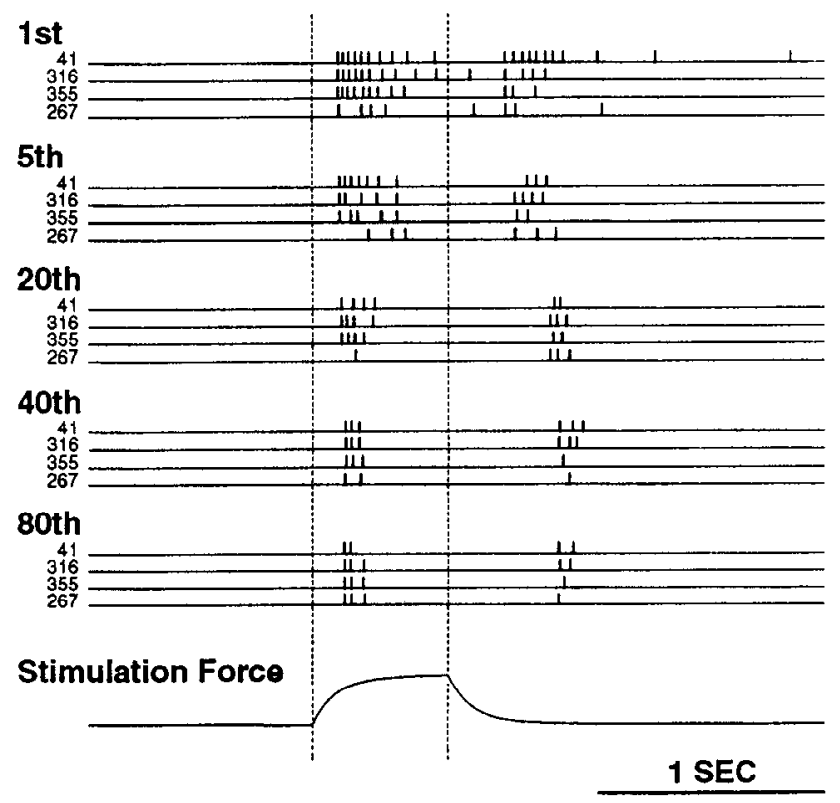

Figure 4. Raster diagram of the spike activities of the first four cells in group I from Figure 2. The onset and offset responses of these neurons are most clearly seen after habituation. The $1 \mathrm{st}, 5 \mathrm{th}, 20 \mathrm{th}, 60 \mathrm{th}$, and 80 th habituation trials are shown. The bottom trace is the stimulus force recorded with a force transducer in series with the mechanical toucher. The dashed vertical lines represent the onset and offset of the current pulse driving the toucher motor controller.

grams emphasize the dramatic differences in response patterns of the six groups. The neurons in group I gave a brief response (mainly at the onset and offset of the stimuli). This response partially habituated and then was maintained at a relatively constant level. Group II consists of cells with a sustained response to the stimulus. Like group I neurons, the habituation was only partial. The neurons in group III had a similar sustained pattern in control trials, but ceased to respond early in the habituation process. Group IV contains the few detected cells (one to three per preparation) whose activity was inhibited by the stimulus. These cells were apparently less inhibited after habituation (see also Fig. 5). Group $V$ includes neurons that were relatively weakly activated by the stimuli and did not fit into one of the first four groups. Cells that did not respond to the stimuli are in group VI.

A quantitative display of the results of clustering can be visualized using a two-dimensional plot (Fig. 3). Since the grouping was based on the stimulus-response relationship and the effects of habituation, we chose axes representing these features. For the $\mathrm{x}$-axis we chose a parameter that measures the response in the first 1024 data points immediately after the stimulus, adjusted for the prestimulus spike rate and normalized relative to the total amount of spike activity. This parameter is sensitive to the response patterns of the cells. The parameter used for the $y$-axis measured the degree of habituation. Each neuron from groups I-III of Figure 2 is plotted as a point in Figure 3. Although the neurons are not ideally clustered, cells in the three groups are separated, thereby providing an independent support for the grouping of Figure 2. If group IV-VI neurons were plotted in this figure, group IV neurons would be on the negative side of the $\mathrm{x}$-axis, many in group $\mathrm{V}$ and VI would be in the area between 


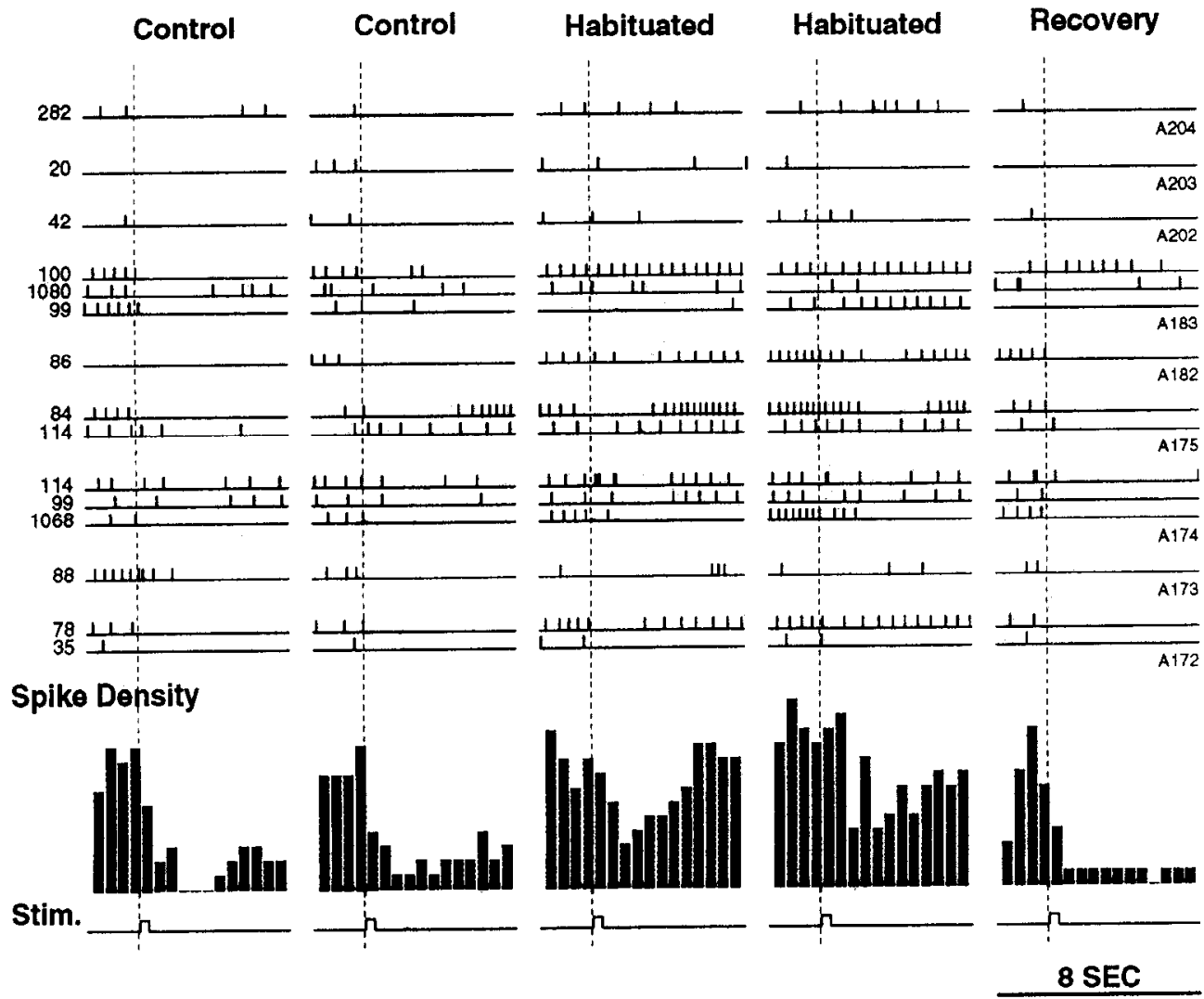

Figure 5. Raster diagrams of the activity of inhibited neurons (group IV) from all nine experiments $(t o p)$ and the corresponding spike density (spike counts per $812 \mathrm{msec}$ across all the cells in each panel) histogram (bottom). The inhibitory effect of the stimulus was rapid; habituation diminished the inhibitory effect of the stimulus on these neurons. groups II and III, while the remainder would be widely scattered. Thus, these two parameters are not adequate to separate all six groups. We presume that with additional parameters (axes), all groups would separate well although the result would be difficult to visualize.

The neurons from all nine habituation experiments can be clustered into the same six groups although animal-to-animal variation exists. For example, in some experiments, the offset response of group I neurons was much less prominent than the onset response. In addition, in all the experiments there were cells that were difficult to categorize. Cells with a borderline response pattern were either assigned to one of the groups to which they appeared to belong or included in group V. However, even though there is variability in such a grouping, the six groups with the characteristics described in the previous paragraph are distinct in all nine animals.

In the nine experiments, an average of $70 \pm 8$ neurons were detected: $11 \pm 1 \%$ were in group I, $15 \pm 2 \%$ in group II, $13 \pm$ $2 \%$ in group III, $3 \pm 1 \%$ in group IV, $26 \pm 2 \%$ in group $\mathrm{V}$, and $32 \pm 3 \%$ in group VI (mean \pm SEM)

To determine if any of the neurons in the preparation shown in Figure 2 were primary sensory neurons, an additional optical recording was made after bathing the ganglion in a high-magnesium/low-calcium seawater (see Materials and Methods) that blocks chemical synaptic transmission. All the neuronal responses to touch were eliminated. The responses recovered when seawater was returned. This result was also obtained in two additional experiments with high $\mathrm{Mg}^{2+} / \mathrm{low} \mathrm{Ca}^{2+}$ and in three experiments using a $\mathrm{Co}^{2+}$ solution $(460 \mathrm{~mm} \mathrm{NaCl}, 10 \mathrm{~mm} \mathrm{KCl}$, $1 \mathrm{mM} \mathrm{CaCl}_{2}, 10 \mathrm{mM} \mathrm{CoCl}_{2}, 55 \mathrm{mM} \mathrm{MgCl}_{2}$, and $10 \mathrm{~mm}$ Tris$\mathrm{HCl}, \mathrm{pH} \mathrm{7.4)}$ to block the synaptic transmission. Thus, it appears that none of the neurons in Figure 2 were likely to be primary sensory neurons.

Although group I neurons are probably not primary sensory neurons, these neurons encode the onset and offset of the sensory stimulus. This result is most clearly seen when the gill withdrawal was habituated. Figure 4 illustrates, on an expanded time scale, the response of the first four neurons from Figure 2. The stimulus force is shown at the bottom. The onset response followed the initiation of the stimulus with a short and consistent delay, while the offset response tended to have an increased delay after habituation. Supporting evidence for the existence of neurons that encode the onset and offset of the stimulus came from separate extracellular measurements from genital and branchial nerves where siphon stimuli of several durations were applied. Units were found that were active at the onset and offset independent of stimulus duration.

The group I neurons had the earliest response to the stimulus. The mean delay between the start of the stimulus and the first spikes in group I neurons was about $180 \pm 20 \mathrm{msec}$ (mean \pm SEM; 9 experiments). The first spikes in group I cells came 360 $\pm 80 \mathrm{msec}$ before the first spikes in group II neurons and 850 $\pm 130 \mathrm{msec}$ before those in group III cells.

In contrast with group I cells, the activity of the neurons in groups II-IV is better correlated (negatively for group IV) with the behavioral output than to the stimulation in terms of the time course of response. This correlation was also observed in a recording made when the habituating stimulus happened to occur during a spontaneous gill contraction (data not shown). Most cells in groups II and III, but not in group I, were active during the spontaneous contraction even though their response to siphon touch was already habituated. Further, in this instance 


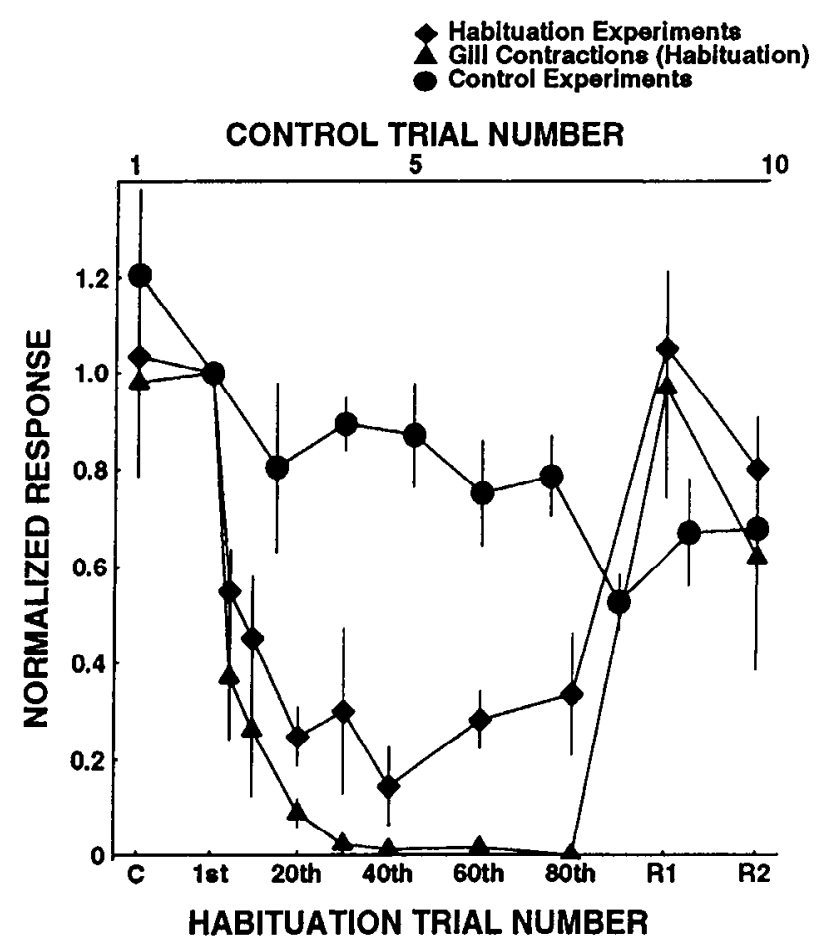

Figure 6. Plots of the normalized overall neuronal response (diamonds) and the corresponding gill contractions (triangles) from the nine habituation experiments, and the overall neuronal response from five control experiments (circles). The neuronal response for an individual neuron was defined as the difference in spike density before and after the stimulus. The overall neuronal response for a trial is the sum of the responses of individual neurons. Because the total number of the neurons detected $(70 \pm 8)$ is different from experiment to experiment due to variability in the preparation and effectiveness of the staining procedure, the overall responses were normalized to the response from the second (control) trial for each experiment. The means and the SEM of the normalized response were calculated across all nine experiments and are plotted here. The gill contractions were measured as the size of the signals extracted from the videotape recordings.

the inhibited neurons (group IV) were silent instead of firing constantly as they often do in the habituated state (see below).

The effects of habituation on inhibited neurons are illustrated in Figure 5, which combines the examples from all nine preparations. The histogram on the bottom represents the spike density summed over all the cells in the nine experiments in each trial (vertical panel). Although there is trial-to-trial and animal-to-animal variability, it seems clear from the histograms that the inhibitory effect of the stimulus is relatively rapid and that habituation decreased the inhibitory effect. There is no sign of an excitatory response preceding the inhibition.

\section{Habituation effects on the overall neuronal response}

We measured the difference in spike density before and after the stimulus for each neuron in all of the experiments. Figure 6 plots this overall neuronal response from the nine habituation experiments and the five control experiments. For the habituation experiments the neuronal responses (diamonds) and the corresponding gill contractions (triangles) are plotted against the habituation trial number. For the control experiments, the overall neuronal responses (circles) are plotted using the upper horizontal axis chosen so that the habituation and the control data fit on the same plot. The change in neuronal response during

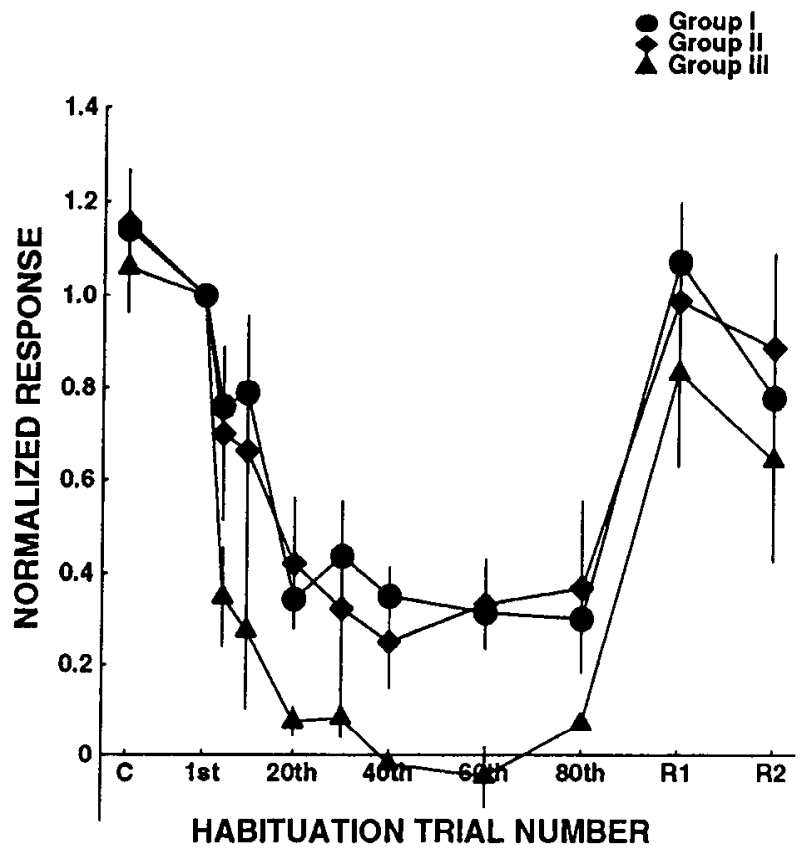

Figure 7. Plots of the neuronal response in group I (circles), group II (diamonds), and group III (triangles) from nine habituation experiments. Negative responses (seen in habituated group III neurons) result from random background activity before the stimulus. These negative response are not significantly different from zero.

the habituation process is well correlated with the change in behavior. Both drop rapidly at the beginning of the habituation. The neuronal response reaches a plateau of $20-40 \%$ of the control response level while the gill contraction decreases to near zero.

There is a slight but statistically significant decrease in the overall neuronal response of the control experiments. Two possible causes might account for this decrease. First, a slight habituation might have occurred even with $15 \mathrm{~min}$ ISIs. Second, photodynamic damage and/or dye bleaching might have led to a reduced response. However, the fact that the habituated group recovers to control levels argues against this second explanation.

Plots of the neuronal response for the individual groups (IIII) are shown in Figure 7. Group III neurons habituate more rapidly than those in groups I and II. Clearly, for both group I (circles) and group II (diamonds), the response reaches a plateau ( $30-40 \%$ of control) that is significantly greater than zero, while the response decreased to near zero for group III (triangles).

\section{Spatial distribution of the neurons}

To determine whether the neurons in each group were anatomically clustered or spread throughout the ganglion, we plotted the spatial distribution of neurons in groups I-IV from all nine experiments. The results are shown in Figure 8. Most of the group IV neurons were found in the right upper quadrant. Group II neurons were more concentrated along the edge of the left upper quadrant near the entrance of the siphon nerve. The cells in groups I and III were more evenly spread throughout the ganglion. Even though there are variations in the locations of neurons from animal to animal and the ganglion was not positioned at exactly the same place in relation to the photodiode array from experiment to experiment, the spatial distribution of individual groups seems to have common features across 
animals. However, the location of the signals on the array only provides information about the location of the cell in the $\mathrm{x}, \mathrm{y}-$ plane; we do not have information about the location of the neurons in the z-axis. In principle, this information might be obtained by making simultaneous optical measurements with two orthogonal imaging systems and arrays. In the absence of $\mathrm{z}$-axis information the source of the signals can be correlated with images of neurons only in exceptional cases (e.g., London et al., 1987).

\section{Discussion}

We used voltage-sensitive dye recordings to study the effects of habituation on neuronal activity in the Aplysia abdominal ganglion. The ability of this technique to monitor the activity of a large number of neurons has led to the finding that there are several groups of neurons that are defined by their response to siphon stimulus and habituation training. These include the discovery of two new response types: group I neurons that mainly encode the onset and offset of the sensory stimulus, and group IV neurons that are inhibited by the stimulus. We are not aware of previous reports of neurons that respond in these ways to a light touch.

Because the activity of the group I neurons (as well as all other ncurons) was blocked when the ganglion was bathed in synaptic blockers, it seemed unlikely that they were primary sensory neurons. In addition, their size, spatial distribution in the ganglion, and the facts that they respond to light touch and their response habituates, all suggest that they are not like the LE, rLE, RE, or RF (Byrne et al., 1974, 1978a,b; Dubuc and Castellucci, 1990) sensory neurons. However, the group I neurons do respond to touch in a pattern that is similar to that found when leech T-cells are activated by substantially suprathreshold tactile stimulation (7 gm; Nicholls and Baylor, 1968) and to that when sensory neurons in the Aplysia pleural ganglia are stimulated with a moderately intense touch (Billy and Walters, 1989). We conclude that group I neurons are probably not primary sensory neurons. However, their response suggests a possible response pattern for the as yet unidentified sensory neurons for light touch.

The fact that we did not detect primary touch sensory neurons in our optical recording may mean that the cell bodies of these sensory neurons are in the periphery rather than in the abdominal ganglion. Alternatively, if the cell bodies are in the ganglion, they might be small and thereby generate optical signals with a signal-to-noise ratio of less than 1.

The group I type response can also be seen in the activity recorded from both genital and branchial nerves, suggesting that the neurons responsible for these nerve responses might be motor neurons triggering the initial gill movement or neurons transmitting information to the PNS. Other members of group I might be interneurons that process the stimulus input and provide output to other interneurons and motor neurons. The plateau that the response of group I neurons reaches after habituation suggests that the nervous system has a mechanism whereby habituation reduces the neuronal response to the point where the system produces no gill contraction while remaining aware of the peripheral input. Both group II and group III neurons have sustained responses. Their firing patterns may help determine the duration, amplitude of the gill contraction and quality (Leonard et al., 1989).

An inhibitory response to the touch may be more widespread in the abdominal ganglion than the fraction of inhibited neurons
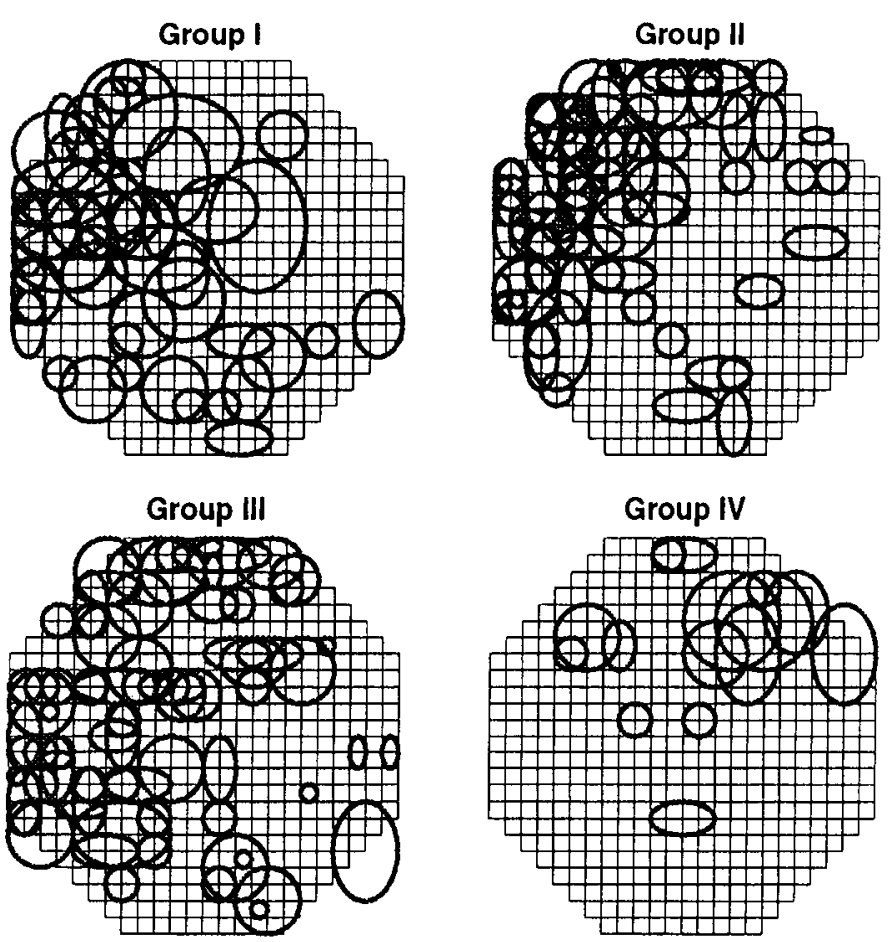

Figure 8. Spatial distribution of neurons in groups I-IV from all nine experiments. The neurons in group IV are mainly found in the upper right quadrant. The neurons in group II are concentrated on the upper left edge. The cells in groups I and III are more evenly distributed. The plotting program used the number and locations of diodes with signals larger than $50 \%$ of the largest signal to determine the size and position of the ellipses used to represent cell bodies.

(group IV) we detected. Aersten and Gerstein (1985) noted that detection of inhibitory responses in measurements of spike activity depends on the level of spontaneous background activity in the prestimulus period. When the background spike activity is low, the detectability of inhibition is also low. Less than $5 \%$ of the Aplysia neurons were spontaneously active in the prestimulus period. Thus, for the majority of Aplysia neurons it is not possible to detect an inhibitory effect of the stimulus from measurements of spike activity. The number of inhibited neurons might be quite large.

Some of the neurons that are inhibited by the stimulus (Fig. 5) may receive this inhibition directly from sensory axons. While many identified inhibitory neurons have been described in the abdominal ganglion (Hawkins et al., 1981), almost all receive excitatory inputs from LE sensory neurons and, in turn, inhibit other neurons excited by LE neurons. Thus, the followers of these inhibitory neurons are likely to be first excited by sensory neurons and then inhibited. Since inhibition of group IV neurons often occurred rapidly and without antecedent excitation, it raises the possibility that sensory neurons might make inhibitory synapses onto some followers.

The correspondence between the neurons recorded optically and those previously identified by other laboratories is difficult to determine. There are two obstacles. First, it would be very difficult to obtain a comprehensive map of electrophysiologically identified neurons while keeping an intact ganglion in one position. Some identified neurons are not accessible to microelectrode impalement without turning the ganglion over. In addition, because the sheath of the ganglion was kept intact to 
avoid damage to neurons, it was impossible to impale identified cells other than those in the outermost layer. Second, except for a small number of very large neurons, it is not easy to attribute neurons detected in optical recordings to neurons in a photograph of the ganglion. The photograph only has clear images of the neurons on the top surface of the ganglion while the optical recordings detect neurons throughout the thickness of the ganglion (Salzberg et al., 1977). While the optical recordings provide information about neuron positions in the $\mathrm{x}, \mathrm{y}$-plane (object plane of the microscope), there is no information about neuron position in the $\mathrm{z}$-axis (dorsal-ventral axis in these experiments) from individual trials. This information might be obtained from repeated optical recordings at several focal positions, but trialto-trial variability (Wu et al., 1989; Wu, Tsau, Cohen, Tang, Falk, and Hopp, unpublished observations) would complicate the interpretation. Although simultaneous optical and microelectrode recordings have been made from intact leech (Salzberg et al., 1973) and barnacle (Salzberg et al., 1977) ganglia, and also from trypsin- treated Aplysia ganglia (Wu, Tsau, Cohen, Tang, Falk, and Hopp, unpublished observations), an extensive comparison of previously identified neurons and those recorded optically would not be easily achieved.

Nevertheless, our results indicate that the response to habituation in the neurons of the Aplysia abdominal ganglion is far from simple. Both group I and group II neurons maintain a relatively constant response, while the response level of group III neurons decreases to near zero after habituation. Although a decrease in response is found in all three groups, there are differences in the rate of decrease and the plateau of their final response level. It is possible that the diverse expression of habituation can be explained by a simple decrement in synaptic efficacy of the sensory neurons and/or differences in channel type and density among the postsynaptic neurons. The diverse expression of habituation, however, also raises the possibility that several synaptic mechanisms are involved.

While our results show that the activity of many neurons is affected by habituation, they provide no information about which neurons are causally related to the gill withdrawal or its plasticity. On one hand, it is possible that there is a dedicated circuit in the abdominal ganglion that is specific for the gill withdrawal reflex. A number of gill motor neurons and interneurons that synapse on them have been identified (Hawkins et al., 1981; Frost et al., 1988), but the sensory neurons responsible for the response to a light touch have not been identified and there may be additional interneurons as well. On the other hand, it is possible that the gill withdrawal reflex and its habituation are controlled coordinately along with other behaviors by a distributed network (Kien et al., 1992). Many neurons are activated by a light touch that elicits the gill withdrawal reflex (Zecevic et al., 1989; Nakashima et al., 1992). Furthermore, a study of the relationship of the evoked gill withdrawal reflex and the spontaneous gill contraction revealed that there was more than $70 \%$ overlap in the neuronal populations for the two behaviors (Wu et al., 1991). These results support the notion that a distributed network might generate the gill withdrawal reflex. In that case, a large fraction of the optically recorded neurons would be participants in the reflex and its habituation.

\section{References}

Aersten ADMH, Gerstein GL (1985) Evaluation of neuronal connectivity: sensitivity of cross-correlation. Brain Res 340:341-354.

Bailey $\mathrm{CH}$, Chen $\mathrm{M}$ (1988) Morphological basis of short-term habituation in Aplysia. J Neurosci 8:2452-2459.
Billy AJ, Walters ET (1989) Long-term expansion and sensitization of mechanosensory receptive fields in Aplysia support an activitydependent model of whole-cell sensory plasticity. J Neurosci 9:12541262.

Byrne JH (1982) Analysis of synaptic depression contributing to habituation of gill-withdrawal reflex in Aplysia californica. J Neurophysiol 48:431-438.

Byrne J, Castellucci V, Kandel ER (1974) Receptive fields and response properties of mechanoreceptor neurons innervating siphon skin and mantle shclf in Aplysia. J Ncurophysiol 37:1041-1064.

Byrne JH, Castellucci VF, Carew TJ, Kandel ER (1978a) Stimulusresponse relations and stability of mechanoreceptor and motor neurons mediating defensive gill-withdrawal reflex in Aplysia. J Neurophysiol 41:402-417.

Byrne JH, Castellucci VF, Kandel ER (1978b) Contribution of individual mechanoreceptor sensory neurons to defensive gill-withdrawal reflex in Aplysia. J Neurophysiol 41:418-431.

Cash D, Carew TJ (1989) A quantitative analysis of the development of the central nervous system in juvenile Aplysia californica. J Neurobiol 20:25-47.

Castellucci V, Pinsker H, Kupfermann I, Kandel ER (1970) Neuronal mechanisms of habituation and dishabituation of the gill-withdrawal reflex in Aplysia. Science 167:1745-1748.

Coggeshall RE (1967) A light and elcctron microscope study of the abdominal ganglion of Aplysia californica. J Neurophysiol 30:12631287.

Cohen TE, Henzi V, Kandel ER, Hawkins RD (1991) Further behavioral and cellular studies of dishabituation and sensitization in Aplysia. Soc Neurosci Abstr 17:1302.

Dubuc B, Castellucci VF (1990) Receptive fields and properties of a new cluster of mechanoreceptor neurons innervating the mantle region and the branchial cavity of the marine mollusk Aplysia californica. Soc Neurosci Abstr 16:399.

Dudai Y (1989) The neurobiology of memory, pp 49-76. New York: Oxford UP.

Everitt B (1974) Cluster analysis. London: Cox and Wyman.

Falk CX, Wu JY, Cohen LB (1991) Effects of habituation on neuronal activity in the Aplysia abdominal ganglion during the gill-withdrawal reflex. Soc Neurosci Abstr 17:548.

Falk CX, Wu J-Y, Cohen LB, Tang AC, Tsau Y (1992) A study of neuronal response in Aplysia abdominal ganglion during habituation of the gill withdrawal reflex. Soc Neurosci Abstr 18:941.

Frost WN, Clark GA, Kandel ER (1988) Parallel processing of shortterm memory for sensitization in Aplysia. J Neurobiol 19:297-334.

Goldberg JI, Lukowiak K (1984) Transfer of habituation in Aplysia: contribution of heterosynaptic pathways in habituation of the gillwithdrawal reflex. J Neurobiol 15:395-411.

Grinvald A, Manker AA, Segal M (1982) Visualization of the spread of electrical activity in rat hippocampal slices by voltage sensitive optical probes. J Physiol (Lond) 333:269-291

Hawkins RD, Castellucci VF, Kandel ER (1981) Interneurons involved in mediation and modulation of the gill-withdrawal reflex in Aplysia. I. Identification and characterization. J Neurophysiol 45: 304-314.

Kien J, McCrohan CR, Winlow W, eds (1992) Neurobiology of motor programme selection. Oxford: Pergamon.

Kupfermann I, Castellucci V, Pinsker H, Kandel ER (1970) Neuronal correlates of habituation and dishabituation of the gill-withdrawal reflex in Aplysia. Science 167:1743-1745.

Kupfermann I, Pinsker H, Castellucci V, Kandel ER (1971) Central and peripheral control of gill movements in Aplysia. Science 174: $1252-1256$

Lconard JL, Edstrom J, Lukowiak K (1989) Recxamination of the gill withdrawal reflex of Aplysia californica Cooper (Gastropoda; Opisthobranchia). Behav Neurosci 103:585-604.

London JA, Zecevic D, Cohen LB (1987) Simultaneous optical recording of activity from many neurons during feeding in Navanax. $J$ Neurosci 7:649-661.

Lukowiak K (1978) L9 modulation of gill withdrawal reflex habituation in Aplysia. J Neurobiol 10:255-271.

Nakashima M, Yamada S, Shiono S, Maeda M, Satoh F (1992) 448-detector optical recording system: development and application to Aplysia gill-withdrawal reflex. IEEE Trans Biomed Eng 39:26-36.

Nicholls JG, Baylor DA (1968) Specific modalities and receptive fields of sensory neurons in the CNS of the leech. J Neurophysiol 31:740 756. 
Pinsker H, Kupfermann 1, Castellucci V, Kandel ER (1970) Habituation and dishabituation of the gill-withdrawal reflex in Aplysia. Science 167:1740-1742

Salzberg BM, Davila HV, Cohen LB (1973) Optical recording of impulses in individual neurons of an invertebrate central nervous system. Nature 246:508-509.

Salzberg BM, Grinvald A, Cohen LB, Davila IIV, Ross WN (1977) Optical recording of neuronal activity in an invertebrate central nervous system: simultaneous monitoring of several neurons. J Neurophysiol 40:1281-1291.

Trudeau LE, Castellucci VF (1992) Contribution of polysynaptic pathways in the mediation and plasticity of Aplysia gill and siphon withdrawal reflex: evidence for differential modulation. J Neurosci 12: $3838-3848$.
Wu JY, Cohen LB (1993) Fast multisite optical measurement of membrane potential. In: Fluorescent probes for biological function of living cells - a practical guide (Mason WT, ed), in press. London: Academic.

Wu JY, Falk CX, Hopp H-P, Cohen LB (1989) Trial-to-trial variability in the neuronal response to siphon touch in the Aplysia abdominal ganglion. Soc Neurosci Abstr 15:1264.

Wu JY, Cohen LB, Falk CX (1991) Comparison of neuronal activity in the Aplysia abdominal ganglion during respiratory pumping and the gill-withdrawal reflex. Soc Neurosci Abstr 17:549.

Zecevic D, Wu J-Y, Cohen LB, London JA, Hopp H-P, Falk CX (1989) Hundreds of neurons in the Aplysia abdominal ganglion are active during the gill-withdrawal reflex. J Neurosci 9:3681-3689. 